University of Wollongong

Research Online

Faculty of Engineering and Information

Faculty of Engineering and Information

Sciences - Papers: Part A

Sciences

$1-1-2013$

Defect dynamics in polycrystalline zirconium alloy probed in situ by primary extinction of neutron diffraction

Saurabh Kabra

Australian Nuclear Science and Technology Organisation

Kun Yan

University Of Wollongong

David G. Carr

Australian Nuclear Science and Technology Organisation

Robert P. Harrison

Australian Nuclear Science and Technology Organisation

Rian J. Dippenaar

University of Wollongong, rian@uow.edu.au

See next page for additional authors

Follow this and additional works at: https://ro.uow.edu.au/eispapers

Part of the Engineering Commons, and the Science and Technology Studies Commons

Research Online is the open access institutional repository for the University of Wollongong. For further information contact the UOW Library: research-pubs@uow.edu.au 


\title{
Defect dynamics in polycrystalline zirconium alloy probed in situ by primary extinction of neutron diffraction
}

\author{
Abstract \\ After $\alpha+\beta$-zirconium has fully transformed into $\beta$-phase upon heating, the intensities of all $\beta$-Zr Bragg \\ reflections decrease simultaneously as a function of time. It is shown that this effect represents a \\ transition from the kinematic to the dynamic theory of diffraction due to the ever increasing crystal \\ perfection driven by thermal recovery of the system. The best fitting coherent crystallite size of $30 \mu \mathrm{m}$ \\ and other microstructural features are verified by in situ laser scanning confocal microscopy. This effect \\ of primary extinction in neutron diffraction has been employed to further investigate the crystal perfection \\ kinetics. Upon further heating, crystal recovery is identified as a process of dislocation annihilation, \\ suffering from lattice friction. Upon cooling, precipitating $\alpha-Z r$ induces strain into the perfect $\beta$ - \\ crystallites, re-establishing the kinematic diffraction intensities. An Avrami analysis leads to the \\ estimations of nucleation time, consumption of nucleation sites and lower-dimensional growth. Such \\ technique bears great value for further investigation on all metal systems annealed close to the melting \\ temperature. (c) 2013 American Institute of Physics.
}

\section{Keywords}

polycrystalline, dynamics, defect, situ, alloy, zirconium, primary, diffraction, neutron, probed, extinction

\section{Disciplines}

Engineering | Science and Technology Studies

\section{Publication Details}

Kabra, S., Yan, K., Carr, D. G., Harrison, R. P., Dippenaar, R. J., Reid, M. \& Liss, K. (2013). Defect dynamics in polycrystalline zirconium alloy probed in situ by primary extinction of neutron diffraction. Journal of Applied Physics, 113 (6), 063513-1-063513-8.

\section{Authors}

Saurabh Kabra, Kun Yan, David G. Carr, Robert P. Harrison, Rian J. Dippenaar, Mark Reid, and Klaus-Dieter Liss 


\title{
Defect dynamics in polycrystalline zirconium alloy probed in situ by primary extinction of neutron diffraction
}

\author{
Saurabh Kabra, ${ }^{1}$ Kun Yan, ${ }^{1,2}$ David G. Carr, ${ }^{1}$ Robert P. Harrison, ${ }^{1}$ Rian J. Dippenaar, ${ }^{2}$ \\ Mark Reid, ${ }^{2}$ and Klaus-Dieter Liss ${ }^{1, a)}$ \\ ${ }^{1}$ Australian Nuclear Science and Technology Organisation, Lucas Heights, NSW 2234, Australia \\ ${ }^{2}$ Faculty of Engineering, University of Wollongong, Wollongong, NSW 2522, Australia
}

(Received 13 December 2012; accepted 17 January 2013; published online 13 February 2013)

\begin{abstract}
After $\alpha+\beta$-zirconium has fully transformed into $\beta$-phase upon heating, the intensities of all $\beta$ - $\mathrm{Zr}$ Bragg reflections decrease simultaneously as a function of time. It is shown that this effect represents a transition from the kinematic to the dynamic theory of diffraction due to the ever increasing crystal perfection driven by thermal recovery of the system. The best fitting coherent crystallite size of $30 \mu \mathrm{m}$ and other microstructural features are verified by in situ laser scanning confocal microscopy. This effect of primary extinction in neutron diffraction has been employed to further investigate the crystal perfection kinetics. Upon further heating, crystal recovery is identified as a process of dislocation annihilation, suffering from lattice friction. Upon cooling, precipitating $\alpha-\mathrm{Zr}$ induces strain into the perfect $\beta$-crystallites, re-establishing the kinematic diffraction intensities. An Avrami analysis leads to the estimations of nucleation time, consumption of nucleation sites and lower-dimensional growth. Such technique bears great value for further investigation on all metal systems annealed close to the melting temperature. (C) 2013 American Institute of Physics. [http://dx.doi.org/10.1063/1.4790177]
\end{abstract}

\section{INTRODUCTION}

Zirconium based alloys, including $\mathrm{Zr}-2.5 \mathrm{Nb}$ (mass $\%$ ), are widely used in the nuclear industry with applications ranging from tubing and pressure vessels to fuel cladding. ${ }^{1,2}$ The reason for this success relates to the right combination of strength, ductility, corrosion resistance and neutron economy, offered by these alloys. Although they have been extensively studied and engineered over the last four decades, the renewed interest in nuclear energy ${ }^{3,4}$ and quest for fourth generation reactors and fusion based technology ${ }^{5,6}$ has led to accelerated research on these materials. New insights about these alloys are being revealed through use of advanced neutron and synchrotron X-ray diffraction based techniques, ${ }^{7,8}$ which have come of age lately. ${ }^{9-11}$

At room temperature, $\mathrm{Zr}-2.5 \mathrm{Nb}$ exists as a hexagonal phase $($ hcp $\alpha$ ) with a small volume fraction of cubic (bcc $\beta$ ) phase which differs slightly in compositions. ${ }^{9,12}$ An $\alpha$ to $\beta$ phase transformation takes place upon heating in the range $\mathrm{T}_{\beta \mathrm{s}}=1033 \mathrm{~K}$ to $\mathrm{T}_{\beta \mathrm{f}}=1133 \mathrm{~K} .{ }^{13}$ Above the $\beta$-transus temperature $\mathrm{T}_{\beta \mathrm{f}}$, only the bcc phase with a uniform composition remains in solid solution. A detailed study on the interplay between the different phases and their compositions upon heating has been published in Ref. 9. In the present paper, we discuss the high temperature $\beta$-phase at and above the phase transformation completion temperature, $\mathrm{T}_{\beta \mathrm{f}}$. Here, we report some microstructural characteristics of this $\beta$ phase as inferred from in situ both high-temperature neutron diffraction and laser scanning confocal microscopy.

After the single $\beta$-phase has been reached, in situ neutron diffraction shows that upon heating further above $\mathrm{T}_{\beta \mathrm{f}}$,

\footnotetext{
a) Author to whom correspondence should be addressed. Electronic addresses: liss@kdliss.de and kdl@ansto.gov.au.
}

all $\beta$-peak intensities drop drastically, down to $\sim 25 \%$ for some peaks. We attribute these drops in intensity to the decrease in lattice imperfections, such as strain fields and the mosaic structure of the crystalline lattice of the $\beta$ phase, corresponding to a transition from the kinematic to the dynamic theory of diffraction. ${ }^{14}$ Upon cooling, the peaks recover in intensity as the $\alpha$-phase nucleates, and thus introduces distortions into the $\beta$ phase crystallites. Extinction calculations using the dynamical theory of diffraction show that the drop in peak intensity corresponds to a coherently diffracting length of $\sim 30 \mu \mathrm{m}$, which is then the subgrain size in the $\beta$ crystallites. The analysis provides a novel method to characterize crystallite perfection and its kinetics as a function of temperature and time in this and similar transformation processes.

\section{EXPERIMENTAL}

\section{A. Neutron diffraction}

The alloy used in this study has a nominal composition of $\mathrm{Zr}-2.5 \mathrm{Nb}$ (mass \%). The exact composition is reported in Ref. 9. Cylindrical samples were worked out using electrical discharge machining from extruded billets with the cylindrical axis parallel to the extrusion direction. The samples were nominally $5 \mathrm{~mm}$ in diameter and $20 \mathrm{~mm}$ in height.

Neutron diffraction measurements were carried out on the high intensity diffractometer WOMBAT ${ }^{15}$ at the Australian Nuclear Science and Technology Organisation (ANSTO). The WOMBAT instrument uses monochromatic neutrons and is equipped with a two-dimensional, position sensitive area detector. A Ge-511 monochromator was used to obtain an incident beam of wavelength $\lambda=1.668 \AA$ and corresponding wave number $\mathrm{k}=3.767 \AA^{-1}$. The diffracted 
neutrons were collected on the cylindrical detector which subtends an angle of $120^{\circ}$ on the sample in the diffraction plane and $\sim 20^{\circ}$ in the vertical, out of plane direction.

The WOMBAT instrument is equipped with a vacuum furnace which was used to heat the sample up to $1473 \mathrm{~K}$, while diffraction data were recorded in situ. The 2D diffraction patterns were collected every $60 \mathrm{~s}$ as the sample temperature was ramped up and then down. With the high flux on the WOMBAT instrument, one minute patterns provided sufficient statistics, once the data were integrated azimuthally along the diffraction rings, into $1 \mathrm{D}$ bins depending on diffraction angle $2 \theta$. Instrument independent reciprocal space coordinates $\mathrm{Q}=4 \pi / \lambda \sin (\theta)$ were calculated from the $2 \theta$ value of each bin and will be used throughout the rest of the paper. Two different heating rates, namely $\sim 2 \mathrm{~K} / \mathrm{min}$ and $\sim 20 \mathrm{~K} / \mathrm{min}$ were used on two different specimens. A C-type thermocouple was used close to the sample surface to read and control the temperature.

\section{B. Confocal microscopy}

In situ laser scanning confocal microscopy (LSCM) experiments were undertaken in order to characterize the surface morphologies during the $\alpha$ to $\beta$ to $\alpha$ phase transformation during one heating and cooling cycle. LSCM is an ideal way to optically image surfaces at high temperatures as the infrared radiation from the hot sample surface is discriminated by the confocal pin hole. This area is in sharp focus and the pinhole is scanned with respect to the sample in order to obtain a full frame image of the object. Data were recorded continuously at a frame rate of $30 \mathrm{~s}^{-1}$ as the sample was heated with infrared lamps, located below the sample crucible. Argon gas continuously flushed through the sample chamber in order to minimize surface oxidation at high temperatures. The temperature was recorded using a thermocouple, located on the sample crucible. Details of the technique can be found in Refs. 16-18.

\section{RESULTS AND DISCUSSION}

Neutron diffraction patterns collected as a function of time have been summarized in the 2D plot in Figure 1(a). The gray scale (color online) values represent intensity as a function of scattering vector $\mathrm{Q}$ on the abscissa and time on the ordinate. This diagram represents a series of conventional powder diffraction patterns, as given in the snapshots of Figures 1(b) and 1(c), stacked on top of each other in time, while the sample temperature is varied as represented in the diagram to the right. The present work is focused on the $\alpha \leftrightarrow \beta$ phase transition and the behavior of the $\beta$ phase at high temperatures, therefore data at lower temperatures (below $923 \mathrm{~K}$ ) have been omitted and were already presented elsewhere. ${ }^{9,12}$

Diffraction peaks of the $\alpha$ and $\beta$ phases were Rietveld refined (Figs. 1(b) and 1(c)) and are indexed using tick marks at the top of Fig. 1(a). The lower part of the plot shows that the starting phase consists almost exclusively of the $\alpha$ phase. ${ }^{9,12}$ As the specimen is heated above $\mathrm{T}_{\beta \mathrm{s}} \sim 1033 \mathrm{~K}, \alpha$ peaks disappear and $\beta$ peaks strengthen in intensity, according to their momentary phase fraction. One particular observation from this plot is the significant shift in position of the $\beta$ peaks which is of chemical origin, as explained in detail in

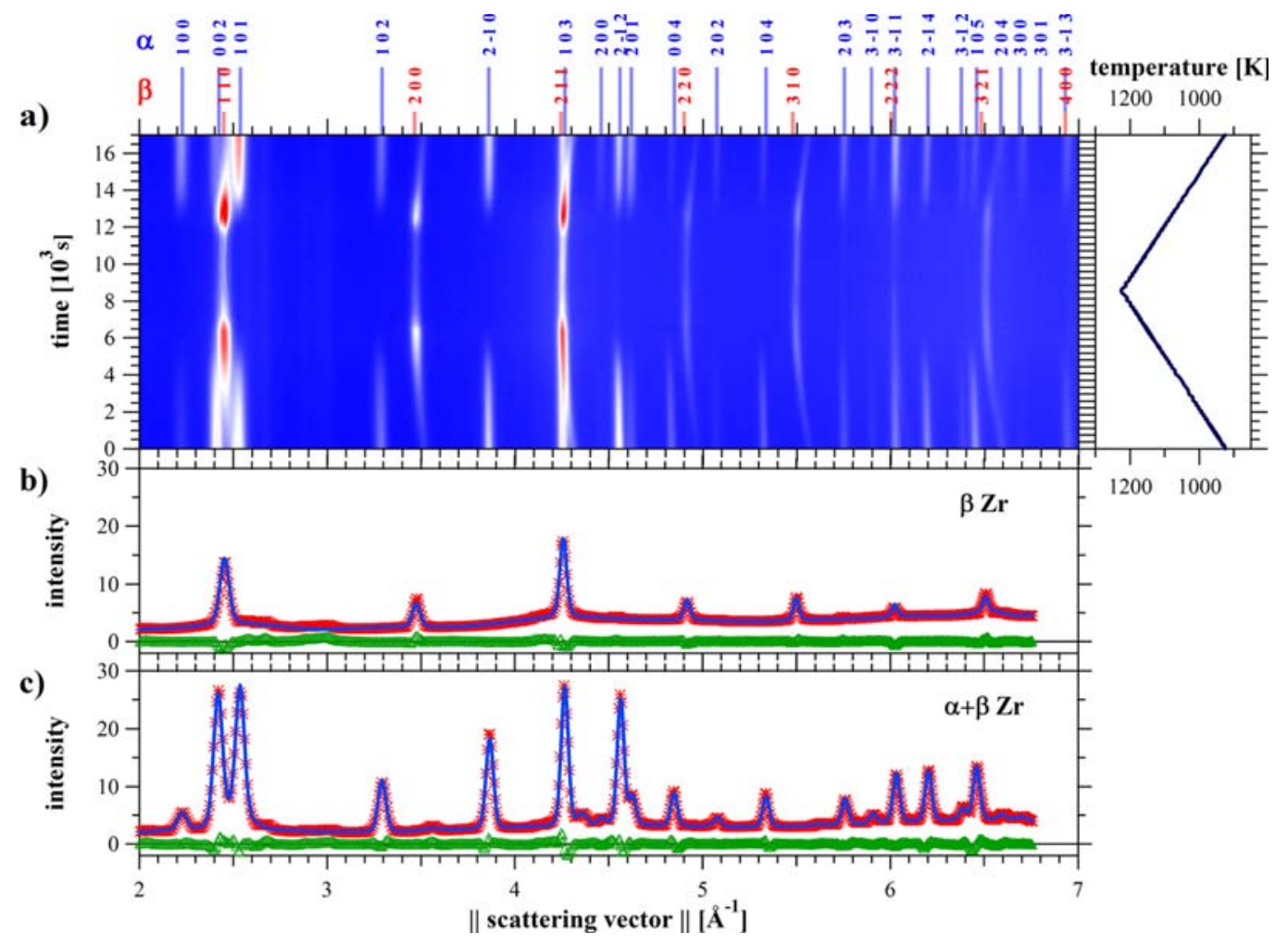

FIG. 1. (a) Neutron diffraction patterns from $\mathrm{Zr}-2.5 \mathrm{Nb}$ as a function of time while temperature is ramped; (b) and (c) Rietveld refinements at snapshots in the $\beta$ and $\alpha+\beta$ phase fields, respectively. 
Refs. 9 and 12. The $\alpha$ phase has been fully transformed to the $\beta$ phase at $\mathrm{T}_{\beta \mathrm{f}} \sim 1123 \mathrm{~K}$ and no further change of peak intensity is expected. However, further heating and passing time shows a drastic drop in all $\beta$ peak intensities up to the maximum temperature of $1223 \mathrm{~K}$, and even during the early stages of subsequent cooling. This drop in all peak intensities is the main focus of this paper and will be discussed in detail. As the specimen is cooled, all peaks start to reestablish intensity at $\mathrm{T}_{\beta \mathrm{f}} \sim 1123 \mathrm{~K}$ and continue to do so until the original full intensity is restored at $\sim 1063 \mathrm{~K}$, corresponding to the $100 \%$ of $\beta$-phase. Upon further cooling, the $\beta$ phase transforms back into the $\alpha$ phase decreasing $\beta$-intensities according to the phase fraction. Notably, the relative intensities of the different reflections of the $\alpha$ phase are different to the starting $\alpha$ phase peak intensities, which we attribute to a change in texture of the $\alpha$ phase resulting from the strong orientation relation between both phases. ${ }^{10,11,19}$

Figures 1(b) and 1(c) show Rietveld fits for the $\alpha$ and $\beta$ phases, respectively. Some regions of the reciprocal space between $2.5 \AA^{-1}$ and $3.0 \AA^{-1}$ show small peaks which could not be fit. These are artifacts from the sample environment used in the experiment and have been ignored in subsequent analysis.

The $\beta$ phase peak intensities were further analyzed individually. The intensities of the major $\beta$ peaks were integrated over a small Q-range (so as to avoid overlap with other peaks) at each temperature. The background in this range was fitted with a second order polynomial and subtracted from the summed intensity. All peak intensities have been normalized to the intensities at $1150 \mathrm{~K}$. In Figure 2(a), the intensities extracted in this way have been plotted as a function of temperature, during heating and subsequent cooling.
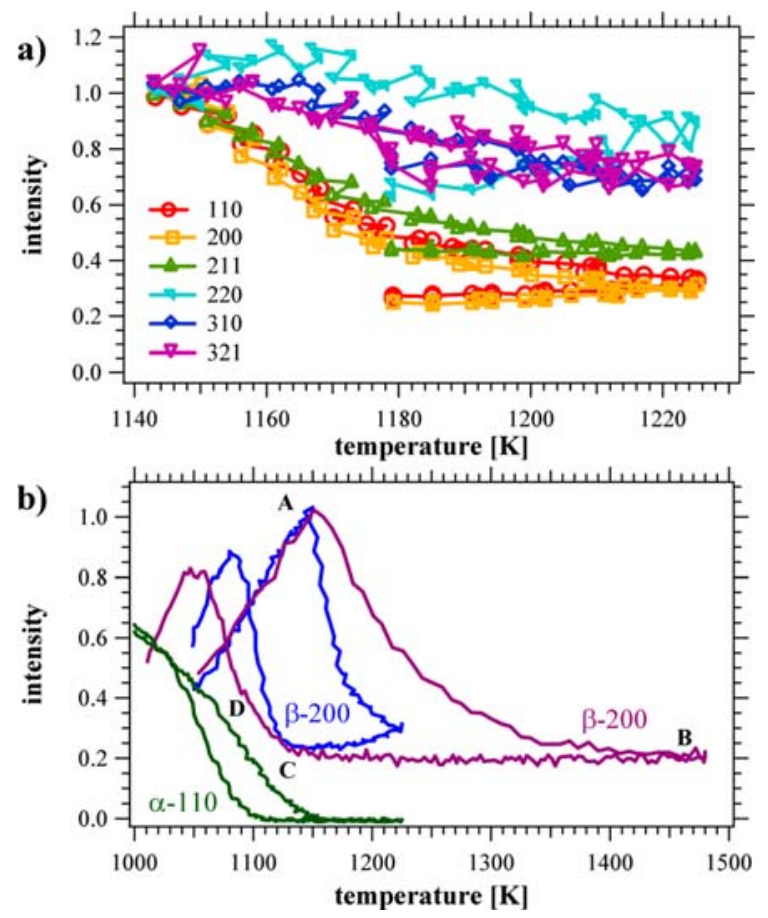

FIG. 2. Behavior of the normalized intensities of $\beta$-Zr reflections (a) and selected reflections (b) for 2 different heating ramps. The features at A,B,C,D are described in the text.
As seen from the plot all the major peak intensities decrease simultaneously. The lower indexed peaks (at lower Q) 110, 200, 211 show the most significant drop in peak intensities ( $\sim 55-75 \%)$, while the drops in the higher Q peaks are in the range of $10-30 \%$. Notably, the high Q peaks show more scatter in the data because the absolute intensities are lower. The simultaneous drop in all peak intensities rules out any change in texture or grain orientation, which might have occurred due to grain growth at these high temperatures. A significant change in texture would have meant that at least some of these peaks would become stronger as those orientations would fall into the diffraction condition. Moreover, texture can be ruled out because multiple reflections like 110 and 220 show huge differences in peak intensity behavior. A change in texture would mean that these reflections change by a similar relative amount.

The simultaneous drop in all intensities and their Q dependence points to a diffraction related effect. For this reason the effect of Debye-Waller factor was considered. In the absence of published Debye-Waller factors for cubic $\mathrm{Zr}$, we used the published values for $\mathrm{Nb},{ }^{20}$ which is the next element from $\mathrm{Zr}$ in the periodic table, to simulate peak intensity drops as a function of temperature, Figure 2(b). There are two notable points here: first, the Q-dependence of intensity drops is opposite of that found in our data, i.e., high $\mathrm{Q}$ intensities drop most and low $Q$ the least in case of Debye-Waller effect; and second, the maximum intensity drop would be $\sim 5 \%$ at $1223 \mathrm{~K}$ which is an order of magnitude less than the drops found in some of the experimental data. Therefore, the Debye-Waller effect can be disregarded.

The $\beta 200$ peak intensities, extracted as described above, from both samples heated at $2 \mathrm{~K} / \mathrm{min}$ and $20 \mathrm{~K} / \mathrm{min}$ have been plotted in Figure 2(b). Also plotted is the $\alpha 110$ (三2 $\overline{1} 0$ ) peak intensity for the $2 \mathrm{~K} / \mathrm{min}$ run. For the latter, the drop in $\alpha$ peak intensity coincides perfectly with the increase in $\beta$ peak intensity, according to the transforming phase fractions. Upon heating beyond $\mathrm{T}_{\beta \mathrm{f}}$ (point $\mathrm{A}$ ), however, the intensity drops drastically by $\sim 75 \%$ over the next $90 \mathrm{~K}$. Notably, the intensity continues to drop even after the cooling commences, indicating that this process not only depends on temperature but moreover on time.

The $20 \mathrm{~K} / \mathrm{min}$ sample, which was heated further up to $1473 \mathrm{~K}$ shows similar behavior. The increase in $\beta$ intensity up to the $\beta$ finish temperature $\mathrm{T}_{\beta \mathrm{f}}$ (point $\mathrm{A}$ ) was identical for both heating rates. However, the rate $\left(\mathrm{K}^{-1}\right)$ of decrease in intensity beyond point A was lower for the $20 \mathrm{~K} / \mathrm{min}$ sample. The intensity drops in both samples approach the same stable low value asymptotically. The relative behavior of the two samples suggests that the responsible phenomenon is highly sensitive to heating rate and is therefore, time dependent. During cooling down, the $\beta 200$ peak intensities of both samples start to increase at $\sim 1123 \mathrm{~K}$ (point $\mathrm{C}$ ) showing a large hysteresis. The $2 \mathrm{~K} / \mathrm{min}$ sample reestablished the intensity at a faster rate $\left(\mathrm{K}^{-1}\right)$ than the $20 \mathrm{~K} / \mathrm{min}$ sample. In both cases, the maximum reestablished intensity was less than the intensity at point $\mathrm{A}$.

We propose that the observed decrease in intensity upon heating of the $\beta$ phase above the $\beta$ finish temperature is due to primary extinction of the incident neutron beam. This 
occurs because of the decrease in the mosaicity of the individual $\beta$ grains within the polycrystalline material as it is heated. As the dislocations are either annealed out of the material or reconfigure themselves, the dislocation-free subgrains grow in this recovery process. Once this perfect crystal unit attains a size comparable to the extinction length, ${ }^{14,21,22}$ primary extinction becomes significant. As the grains become more perfect, the kinematic condition breaks down and diffraction is dominated by the dynamical theory of diffraction. ${ }^{14,21}$ This results in drop of all intensities as a function of $\mathrm{Q}$, as seen in Figure 2(a). The phenomenon of dislocation movement is consistent with the time dependent intensity behavior. The recovery upon cooling commences approximately at the same temperature for the two specimens, at the first instant of nucleation of the $\alpha$ phase. The profuse nucleation of the $\alpha$ phase within $\beta$ grains introduces new imperfections and strain gradients related to lattice mismatch. The primary extinction slowly decreases and thus leading to recovery of $\beta$ peak intensities. The commencement of peak intensity recovery is not coincident with the observed increase in the $\alpha$ peak intensity. This is due to a very low volume fraction of $\alpha$, too small for being detected, which, however, is large enough to impose distortions into the $\beta$-phase, restoring the reflectivities of the latter. To resume, upon heating, the $\beta$ intensity decreases due to primary extinction after the lattice-distorting $\alpha$ phase completely dissolved and the $\beta$ lattice recovers, while it increases due to cease of extinction at the nucleation and growth of $\alpha$ phase upon cooling.

The phenomenon of increase in crystal perfection, as observed through the diffraction data is also consistent with the LSCM micrographs shown in Figure 3. The image of the polished surface of the starting material is mainly composed by $\alpha$ phase. Figures 3(b) and 3(c) show the same area at $1146 \mathrm{~K}$ (point A in Figure 2(b)) and $1504 \mathrm{~K}$. In Figure 3(b), the surface is visibly rough after the phase transformation to the $\beta$-phase. The same area in Figure 3(c) shows distinct grain boundaries in a region where none was present at $1146 \mathrm{~K}$. The grain boundary contrast has increased as the dislocations rearrange themselves at these high temperatures, giving rise to more distinctly defined grain boundaries. The observed surface roughness is an artifact from earlier times, while the visible boundaries stem from a re-arranged $\beta$ microstructure. Figure 3(d) shows a different area at $1120 \mathrm{~K}$ while the sample is cooled back from a high temperature of $1673 \mathrm{~K}$. The micrograph clearly shows new $\alpha$ phase nucleating within the $\beta$ matrix. Notably, this nucleation happens at the same temperature as the peak intensity reestablishes. Upon further cooling to $1092 \mathrm{~K}$, as shown in Figure 3(e), one can see a woven microstructure of well-oriented $\alpha$ precipitates within the $\beta$ matrix. The aligned nature of the microstructure undoubtedly shows strict crystallographic orientation relationship between the $\beta$ and the $\alpha$ phase. The two perpendicular elongated grain types are laths of two different variants of $\alpha$ phase growing from a $\beta$ crystal.

\section{DYNAMICAL THEORY OF DIFFRACTION}

The extinction effect can be quantitatively investigated by the formalism of the dynamical theory of neutron diffrac-
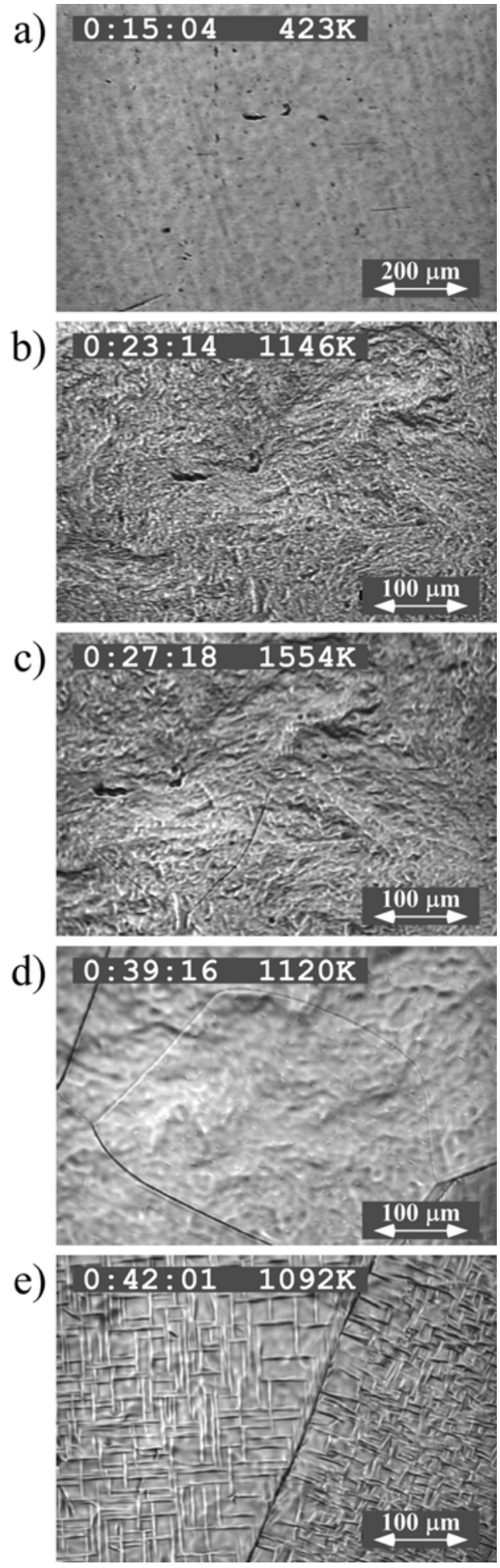

FIG. 3. In situ laser scanning confocal microscopy on $\mathrm{Zr}-2.5 \mathrm{Nb}$ at given time and temperatures of the heating cycle.

tion. ${ }^{14,21}$ In the kinematic theory of diffraction, the integrated Bragg diffracted intensity depends linearly on the volume of the scattering crystal, leading to the paradox that reflected to incident intensity ratios reach infinity with infinitely thick crystals. It has to be considered that crystal layers deeper in the volume of a perfect crystal receive less primary radiation due to extinction — intensity being reflected 
already by layers above. The depth needed at which the primary beam intensity reaches close to nil is called the extinction length and typically ranges a few ten thousand lattice planes and above. Distortions in an imperfect crystal, such as orientation or lattice parameter gradients, however, accept different phase-space elements offered by the incoming radiation source, and sum up to a larger integrated Bragg diffracted intensity. The ultimate limit is the kinematic case unless secondary extinction occurs at much larger length scales.

In dynamical theory, the Fourier compound of the susceptibility $\chi(\mathrm{G})$ at a discrete reciprocal lattice vector $\mathrm{G}=\mathrm{G}_{\mathrm{hkl}}$ with Miller indices $\mathrm{h}, \mathrm{k}, \mathrm{l}$ in reciprocal space $\mathrm{Q}$ derives from the Schrödinger equation to be

$$
\chi(\mathrm{G})=\mathrm{V}(\mathrm{G}) / \mathrm{E}=\left(4 \pi \mathrm{b}_{\mathrm{c}} \mathrm{F}_{\mathrm{hkl}}\right) /\left(\mathrm{k}^{2} \mathrm{~V}_{\mathrm{z}}\right),
$$

where, for neutrons, $V(G)$ is the Fourier component of the Fermi pseudo potential V, E the kinetic energy of the neutron, $b_{c}$ the bound coherent scattering length, $F_{h k l}$ the structure factor, $\mathrm{k}$ the wave number and $\mathrm{V}_{\mathrm{z}}$ the volume of the unit cell. Note that the formally same equation denoting the dielectric susceptibility derives from the Maxwell equations for $X$-rays, where $b_{c}=r_{e} \cdot f(G) \cdot C$ is formally replaced by the classical electron radius $r_{e}$ times the form factor $f(G)$ times a polarization factor $\mathrm{C}$.

For bcc structured material, $\mathrm{F}=2$ for allowed reflections and $\mathrm{V}_{\mathrm{z}}=\mathrm{a}^{3}$ is given by the lattice parameter $\mathrm{a}=3.602 \AA$ of the regarded composition at $1273 \mathrm{~K},{ }^{9}$ while the average neutron scattering length for $\mathrm{Zr}-2.5 \mathrm{Nb}$ (mass \%) $\equiv \mathrm{Zr}-2.46 \mathrm{Nb}$ (at. \%) results with $b_{c, Z r}=7.16 \mathrm{fm}$ and $b_{c, N b}=7.054 \mathrm{fm}^{23,24}$ to $b_{c}=7.157 \mathrm{fm}$, and thus $\chi(\mathrm{G})=2.713 \cdot 10^{-6}$.

The so-called Pendellösung period $\Delta_{0}$, which equals the extinction length to $99.6 \%$ reflectivity in Bragg geometry, is given through

$$
\Delta_{0}=2 \pi / \mathrm{k}\left(\left|\cos \left(\gamma_{0}\right) \cos \left(\gamma_{\mathrm{G}}\right)\right|\right)^{0.5} /|\chi(G)|
$$

In symmetric Bragg geometry, the geometry factor can be approximated through the Bragg angle $\theta$ by

$$
\left(\left|\cos \left(\gamma_{0}\right) \cos \left(\gamma_{\mathrm{G}}\right)\right|\right)^{0.5} \approx \sin (\theta)
$$

which is the simplest approximation for a crystallite embedded in a polycrystalline matrix. Together with Bragg's law $\sin (\theta)=\mathrm{G} /(2 \mathrm{k})$ this results in

$$
\Delta_{0}=\pi \mathrm{G} / \mathrm{k}^{2} /|\chi(G)|
$$

For our material, this resolves to $\Delta_{0}=8.162 \mu \mathrm{m} \cdot \mathrm{G} / \AA^{-1}$, and values for the first 10 reflections are listed in Table I.

The real space extinction length $\Delta_{0}$ limits the sharpness of a Bragg reflection by a natural line width, obtained by the usual correlation between real and reciprocal space, $\Delta \mathrm{k}=$ $2 \pi / \Delta_{0}$. Employing the logarithmic derivative of Bragg's law,

$$
\Delta \mathrm{k} / \mathrm{k}=\Delta \mathrm{G} / \mathrm{G}-\cot (\theta) \Delta \theta
$$

natural line widths on each, the angular scale $\Delta \theta$ or longitudinal lattice strain $|\varepsilon|=\Delta \mathrm{G} / \mathrm{G}$ can be calculated, setting the
TABLE I. Quantitative numbers for reciprocal lattice vector $G_{h k l}$, Miller indices $\mathrm{h}, \mathrm{k}, \mathrm{l}$, multiplicity $\mathrm{m}$, extinction length $\Delta_{0}$, longitudinal and transverse Darwin widths, $|\varepsilon|$ and $\Delta \theta$, lattice spacing $\mathrm{d}_{\mathrm{hkl}}=2 \pi / \mathrm{G}_{\mathrm{hkl}}$ for the first 10 reflections in $\beta \mathrm{Zr}-2.5 \mathrm{Nb}$ in solid solution at $1273 \mathrm{~K}$.

\begin{tabular}{llllrllll}
\hline \hline $\mathrm{G}_{\mathrm{hkl}}\left(\AA^{-1}\right)$ & $\mathrm{h}$ & $\mathrm{k}$ & 1 & $\mathrm{~m}$ & $\Delta_{0}(\mu \mathrm{m})$ & $|\varepsilon|\left(10^{-6}\right)$ & $\Delta \theta(")$ & $\mathrm{d}_{\mathrm{hkl}}(\AA)$ \\
\hline 2.47 & 1 & 1 & 0 & 12 & 20.1 & 8.28 & 4.93 & 2.55 \\
3.49 & 2 & 0 & 0 & 6 & 28.5 & 5.86 & 2.31 & 1.80 \\
4.27 & 2 & 1 & 1 & 24 & 34.9 & 4.78 & 1.43 & 1.47 \\
4.93 & 2 & 2 & 0 & 12 & 40.3 & 4.14 & 0.99 & 1.27 \\
5.52 & 3 & 1 & 0 & 24 & 45.0 & 3.7 & 0.71 & 1.14 \\
6.04 & 2 & 2 & 2 & 8 & 49.3 & 3.38 & 0.52 & 1.04 \\
6.53 & 3 & 2 & 1 & 48 & 53.3 & 3.13 & 0.37 & 0.96 \\
6.98 & 4 & 0 & 0 & 6 & 57.0 & 2.93 & 0.25 & 0.90 \\
7.40 & 4 & 1 & 1 & 24 & 60.4 & 2.76 & 0.11 & 0.85 \\
7.40 & 3 & 3 & 0 & 12 & 60.4 & 2.76 & 0.11 & 0.85 \\
\hline \hline
\end{tabular}

other one to zero. The values of these so-called Darwin widths are added to Table I and show that strain sensitivity on an ideal crystal, with a perfect incoming beam, lies in the microstrain regime while angular sensitivity for mosaic spread measures on the scale of an arc second.

Therefore, the transition from the dynamic to the kinematic theory of diffraction is most sensitive to tiny distortions - on the other hand, primary extinction is barely observed in metals at room temperature as those microstructures are mostly highly distorted. At high annealing temperatures, we demonstrate, crystallites recover towards strain free, ideal crystals and the effects of the dynamical theory of diffraction become applicable.

Lattice gradient induced changes of integrated intensity $\mathrm{R}$ between the kinematic theory and dynamic theory, of a crystal with thickness $D$, with $R_{\text {kin }}=\pi^{2} \mathrm{D} / \Delta_{0}=\pi \mathrm{A}$ and $\mathrm{R}_{\text {dyn }}=\pi \tanh \left(\pi \mathrm{D} / \Delta_{0}\right)=\pi \tanh (\mathrm{A})$, respectively, can be described with the theory of diffraction on gradient crystals, first introduced by Taupin ${ }^{25}$ and Takagi, ${ }^{26}$ or by a transfer matrix method formulated by Liss. ${ }^{14}$ Details, however, are not subject of the present study. The dimensionless thickness $\mathrm{A}:=\pi \mathrm{D} / \Delta_{0}$ measures in multiples of the extinction length and represents a natural unit for each reflection. The intensity drop from a fully kinematic scattering crystal to fully dynamic diffraction of an ideal crystal is then given by $R_{\text {drop }}(A)=1-\left(R_{\text {kin }}(A)-R_{\text {dyn }}(A)\right) / R_{\text {kin }}(A)=\tanh (A) / A$. This theoretical curve is shown in Figure 4 together with $A$ values for the observed reflections evaluated for the best fitting crystallite size of $30 \mu \mathrm{m}$. The presented evaluation was undertaken upon cooling and the obtained undistorted crystal size matches well the undistorted $\beta$-matrix between the $\alpha$-precipitates upon onset of the phase transformation, as shown in the LSCM micrograph of Figure 3(e). Note that intensity drops for low-order reflections, i.e., at larger A values, match better this simple model than higher indexed ones at smaller A. Lower order reflections possess smaller extinction lengths, such that a residual lattice distortion plays a lesser role, while it is still sampled over a larger length scale of a high-order reflection. Therefore, the condition of a perfect crystal is reached earlier for lower than for higher order peaks, such that the latter deviate towards larger reflectivity. Another complication not further considered here is the 


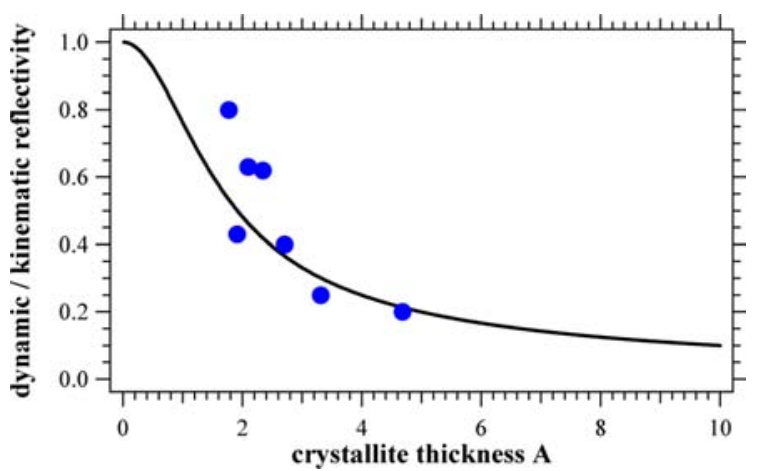

FIG. 4. Ratio between the Bragg reflectivities calculated by the dynamic and the kinematic theory of diffraction, respectively, as a function of crystal thickness normalized to extinction length (continuous line). The dots mark the experimental values of the first 7 reflections at the time of minimal reflectivity. Low-order reflections lie to the right and high order to the left of the distribution.

anisotropy of strain fields with respect to the reciprocal lattice vector, such that different reflections probe for different components of the distortions. It has to be taken into consideration that there might be a lattice orientation dependence of the distortion, such as the orientation of a Burgers vector, which plays a role in evaluating the lattice gradient for a given reflection. Finally, there is a strong relationship between lattice gradients $\mathrm{c}$ and integrated reflectivity $\mathrm{R}$, which is not modeled here, however let's employ a first order approximation, $\mathrm{R} \sim \mathrm{c}$, that reflectivity is linear to the total amount of lattice distortions.

\section{LATTICE DEFECT KINETICS}

The drop of intensity upon heating and its reestablishment upon cooling, Figure 5(a), can be used to obtain information on the kinetics of the underlying crystal lattice. While the present measurements are not ideal for detailed and quantitative studies of the kinetics, they demonstrate the underpinning physics and the way dedicated experiments shall be planned.

The time dependence of the $\beta-\mathrm{Zr} 200$ reflection upon heating is plotted on a double-logarithmic scale in Figure 5(b) and exhibits a straight line over one order of magnitude in time. The intensity, and thus the recovery of crystal perfection follows a strict power law, correlated to the number density $\mathrm{n}$ of dislocations in time $\mathrm{t}$,

$$
\mathrm{n}=\mathrm{n}_{0} \mathrm{t}^{v}
$$

with signatures $\nu=-0.46$ and $\nu=-0.54$ for the slow and the fast heating ramp, respectively. An exponent of $\nu=-0.5$ would be expected in a simple model of dislocation annihilation. Through vacancy diffusion, dislocation move and eventually annihilate spontaneously when they encounter each other with opposite Burgers vectors. Because of their 2 degrees of freedom, the probability of dislocation annihilation is expected to cease by a square root law in time. The process with $\nu=-0.46$ of the gentle heating ramp occurs slower, which we attribute to lattice friction, the pinning and entanglement of dislocations. While easy moving dislocations
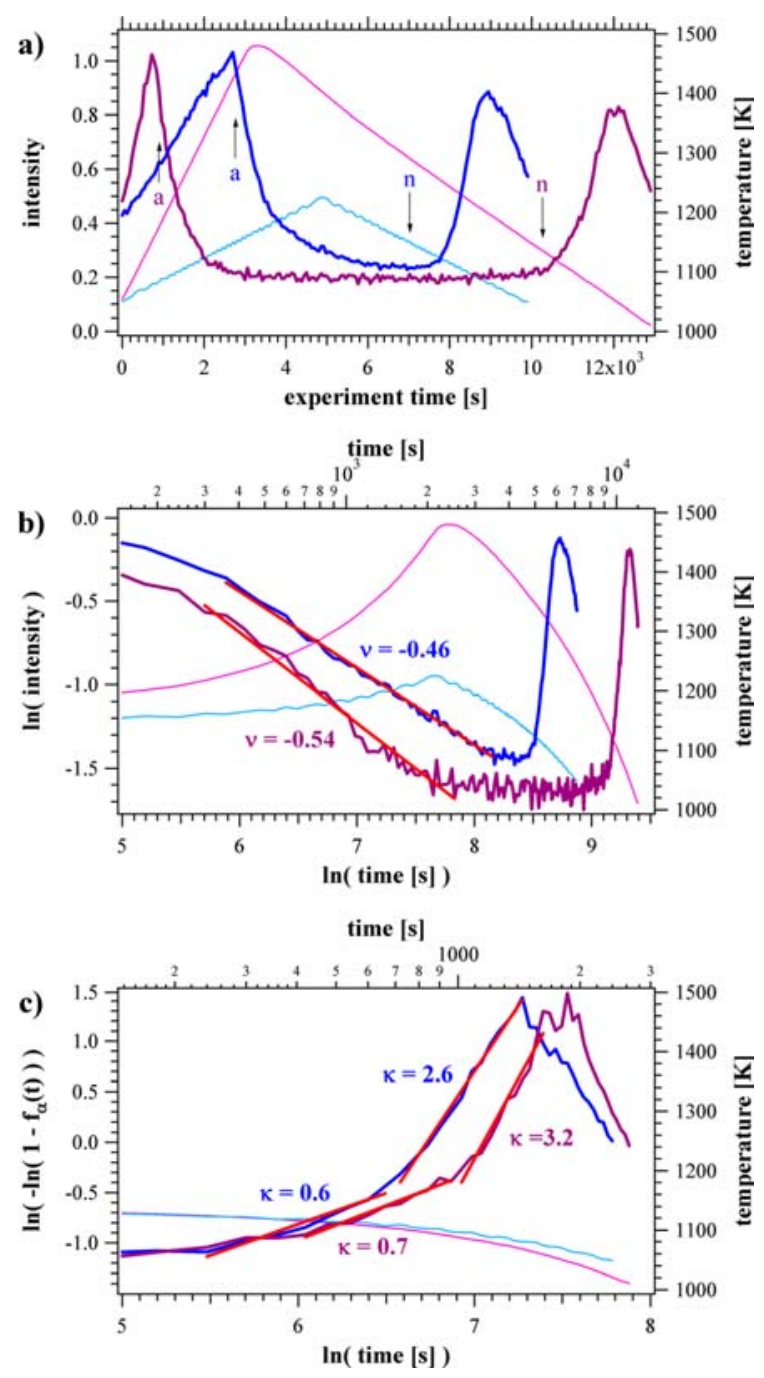

FIG. 5. Time behavior of the $\beta$-200 Bragg reflectivity upon two different heating-cooling cycles (blue, pink). Overall linear plot (a) denoting the zero times for annihilation a and nucleation $\mathrm{n}$ studies in (b) and (c), respectively. The red lines are linear fits to the non-linear plot indicating the slopes nearby.

annihilate first, more and more pinned and motion inhibited dislocations concentrate over time, resulting in a lower absolute value for the exponent. Upon the steeper temperature ramp, $\nu=-0.54$, the annihilation process gets enhanced due to a rapidly raising temperature accompanied by accelerating diffusion. Actually, there is a kink in the curve when changing from heating to cooling, underlining the accelerated, respectively, decelerated annihilation process upon temperature ramping.

Upon cooling, the intensities of the $\beta$-reflections increase again before the phase transforms into $\alpha$-structure and then cease accordingly to the transformation. This is because the $\alpha$-phase nucleates, inducing lattice distortions into the highly perfect $\beta$-phase crystals and destroying gradually the extinction condition, re-establishing the kinematic condition of diffraction. Well before the detection limit of the $\alpha$-reflections, this effect takes place allowing to study the nucleation and growth kinetics of $\alpha$-within $\beta$-phase. For these early stages, we approximate to a linear increase of $\beta$-intensity with amount of $\alpha$-precipitates. For iso-thermal 
holding, nucleation and growth of the precipitating phase fraction $\mathrm{Y}(\mathrm{t})$ at time $\mathrm{t}$ are described by the Avrami equation

$$
\mathrm{Y}(\mathrm{t})=1-\exp \left(-\mathrm{Kt}^{\kappa}\right)
$$

where $\mathrm{K}$ contains the product of nucleation rate and threedimensional growth rate. For an unhindered process, the Avrami exponent $\kappa$ would be $4=1+3$ with respective contributions of nucleation and 3-dimensional growth. Above equation can be written as

$$
\ln (-\ln (1-\mathrm{Y}(\mathrm{t})))=\ln (\mathrm{K})+\kappa \ln (\mathrm{t})
$$

and for, above assumptions, would deliver a linear plot in $\ln (\mathrm{t})$ with slope $\kappa=4$.

Avrami plots of both present cooling cycles are displayed in Figure 5(c). For both cooling rates, we observe non-linear behavior in $\ln (\mathrm{t})$. Qualitatively, we divide the curves into two regions, a nucleation dominated with small slope and a nucleation + growth dominated with large slope, until maximum intensity is recovered and subsequently ceases due to the decrease of the $\beta$-phase. For cooling with $2 \mathrm{~K} / \mathrm{min}, \kappa=0.6$ in the nucleation dominated regime and $\kappa=2.6$ during nucleation and growth. The small Avrami exponent, less than 1, not only suggests the existence of a nucleation time but also reduction of nucleation rate as a function of time, through consumption of nucleation sites. Indeed, it is known that nucleation of $\alpha$-precipitates occurs at grain boundaries, and the sites are consumed once nucleation took place. ${ }^{27}$ Once growth starts, the Avrami exponent jumps by 2 , indicating 2 -dimensional, rather than isotropic growth. This observation matches the knowledge that $\alpha$-precipitates grow in lathes, 2-dimensional manifolds which can also be seen in Figure 3(e) edge-on. The behavior on faster cooling, $20 \mathrm{~K} / \mathrm{min}$, leads to higher Avrami exponents due to the higher thermo-dynamic potential exhibited. Particularly in the growth regime, the curve takes off with ever increasing slope. As the transformation initiates at the same temperature, faster cooling results in more violent processes, expressing in both increasing number of nucleation sites due to growing lattice distortions, as a function of time, not only at the grain boundaries, and increasing growth in the 3rd dimension, i.e., the thickness of the lathes, due to higher thermodynamic potential.

\section{CONCLUSION}

Unconventional behavior of the neutron diffraction intensity of $\beta-\mathrm{Zr}$ phase has been studied in situ, while the $\mathrm{Zr}-2.5 \mathrm{Nb}$ material underwent a heating-cooling cycle. At low temperatures, $\alpha-\mathrm{Zr}$ prevails, which eventually transforms fully into $\beta-\mathrm{Zr}$. After $100 \%$ of $\beta-\mathrm{Zr}$ has been achieved at the $\beta$-transus, $\mathrm{T}_{\beta \mathrm{f}}=1133 \mathrm{~K}$, the intensity of all observed $\beta$-reflections decreases simultaneously as a function of time upon both further heating and subsequent cooling to $\mathrm{T}_{\beta \mathrm{f}}$. At the latter, intensity re-establishes until it diminishes again due to the phase transformation from $\beta$ - to $\alpha-\mathrm{Zr}$. We have shown that the high-temperature decrease in intensity is due to primary extinction as described by the dynamical theory of diffraction. As the underlying crystal lattice recovers upon annealing, crystallites drive more and more towards perfect crystals, and the kinematic theory of diffraction cannot be applied any more. The dependence in momentum transfer, i.e., the intensity decrease for higher order reflections, is fitted with a coherent crystallite size of $30 \mu \mathrm{m}$, which is consistent with in situ laser confocal microscopy studies.

The power law of crystal recovery with signature $\nu \approx-0.5$ corresponds to a mechanism of dislocation annihilation. Smaller absolute $\nu=-0.46$, observed at gentle heating suspects lattice friction by dislocation pinning and entanglement. The recovery process occurs as a function of time, and not primarily of temperature, as it continues on both further heating and subsequent cooling. Only at the time when the $\beta$-transus is reached on cooling, $\alpha-\mathrm{Zr}$ phase nucleates and grows, leading to lattice distortions in $\beta-\mathrm{Zr}$. The intensity increase in $\beta$ - Zr due to lattice distortions impinging from $\alpha-\mathrm{Zr}$ responses very sensitively, well before the $\alpha$-phase can be seen by appearance of its Bragg reflections. Therefore, the intensities of the $\beta$-reflections increase to almost the full amount before they lose again due to the phase transformation. This regime allows to study the nucleation and growth process of $\alpha$-phase within the $\beta$-Zr matrix. Avrami-plots show a distinguished nucleation time in which no or very limited growth occurs. Evidence is given that nucleation sites are consumed, which is consistent with nucleation at grain boundaries. Such nuclei are typically pinned in the tiny, local strain fields existing around each grain boundary before the thermodynamic potential becomes large enough to drive them into growth. The low Avrami-exponents in the growth phase prove of the two- rather than three-dimensional growth, which is identified by the lathes seen by in situ microscopy. Upon violent cooling, $\alpha$-platelets also grow in their thickness leading to a higher Avrami-exponent. Furthermore, the induced strain fields of the precipitating $\alpha$-phase into the $\beta$-phase create further nucleation sites, while the transformation goes off.

The present study is pioneering in interpreting the intensity decrease in single-phase metals at very high temperatures, while recovery of the underlying crystal lattice takes place. This effect is due to primary extinction and is distinct from preferred orientation, texture and Debye-Waller effects. It has been qualitatively seen in other metals, such as $\beta$-titanium and $\delta$-ferrite, and allows to study quantitatively the kinetics of dislocation annihilation as well as the nucleation and growth of precipitates at very high temperatures. A variety of experiments can be envisaged in the future such as obtaining activation energies and information about dynamic behavior during thermo-mechanic plastic flow.

${ }^{1}$ D. G. Cacuci, Handbook of Nuclear Engineering (Springer, Boston, MA, 2010).

${ }^{2}$ C. Lemaignan and A. T. Motta, Zirconium Alloys in Nuclear Applications, in Materials Science and Technology (Wiley-VCH Verlag GmbH \& Co. $\mathrm{KGaA}, 2006)$.

${ }^{3}$ R. W. Grimesand and W. J. Nuttall, "Generating the option of a two-stage nuclear renaissance," Science 329(5993), 799 (2010).

${ }^{4}$ W. J. Nuttall, Nuclear Renaissance: Technologies and Policies for the Future of Nuclear Power (Taylor \& Francis, London, 2010).

${ }^{5} \mathrm{C}$. Forty and P. Karditsas, The Potential Use of Zirconium-Based Alloys in Fusion Power Plants (IEEE, 1997).

${ }^{6} \mathrm{C}$. Forty and P. Karditsas, "Uses of zirconium alloys in fusion applications," J. Nucl. Mater. 283-287, 607-610 (2000). 
${ }^{7}$ M. Kerr, "Mechanical characterization of zirconium hydrides with high energy X-ray diffraction," Thesis, Queen's University, Kingston, 2009.

${ }^{8}$ E. Tulk, M. Kerr, and M. Daymond, Characterization of Hydride Phase Stability in Zirconium Alloys as a Function of Yield Stress with Synchrotron X-ray Diffraction (Minerals, Metals and Materials Society/AIME, Warrendale PA, USA, 2010).

${ }^{9} \mathrm{~K}$. Yan et al., "In Situ Characterization of Lattice Structure Evolution during Phase Transformation of Zr-2.5 Nb," Adv. Eng. Mater 13, 882-886 (2011).

${ }^{10}$ K.-D. Liss, U. Garbe, H. Li, T. Schambron, J. D. Almer, and K. Yan, "In-situ observation of dynamic recrystallization in the bulk of zirconium alloy,” Adv. Eng. Mater. 11(8), 637-640 (2009)

${ }^{11} \mathrm{~K}$.-D. Liss and K. Yan, "Thermo-mechanical processing in a synchrotron beam," Mater. Sci. Eng., A 528(1), 11-27 (2010).

${ }^{12}$ K. Yan, D. G. Carr, S. Kabra, M. Reid, A. Studer, R. P. Harrison, R. Dippenaar, and K.-D. Liss, "In-situ characterization of lattice structure evolution during phase transformation of $\mathrm{Zr}-2.5 \mathrm{Nb}$," World J. Eng. 7(Suppl 2) P422 (2010).

${ }^{13}$ A. Fernandez Guillermet, Z. Metallkd. 82, 478 (1991)

${ }^{14} \mathrm{~K}$. D. Liß, "Strukturelle Charakterisierung und Optimierung der Beugungseigenschaften von $\mathrm{Si}_{1-\mathrm{x}} \mathrm{Ge}_{\mathrm{x}}$ Gradientenkristallen, die aus der Gasphase gezogen wurden," Dissertation, Bibliothek der RWTH Aachen, 1994.

${ }^{15}$ A. J. Studer, M. E. Hagen, and T. J. Noakes, "Wombat: The high-intensity powder diffractometer at the OPAL reactor," Physica B 385-386, 1013-1015 (2006).

${ }^{16} \mathrm{R}$. Dippenaar and D. Phelan, "Delta-ferrite recovery structures in lowcarbon steels," Metall. Mater. Trans. B 34(5), 495-501 (2003).
${ }^{17}$ D. Phelan and R. Dippenaar, "Widmanstätten ferrite plate formation in low-carbon steels," Metall. Mater. Trans. A 35(12), 3701-3706 (2004).

${ }^{18}$ D. Phelan, N. Stanford, and R. Dippenaar, "In situ observations of Widmanstätten ferrite formation in a low-carbon steel," Mater. Sci. Eng., A 407(1-2), 127-134 (2005).

${ }^{19} \mathrm{M}$. Daymond et al., "Texture inheritance and variant selection through an hcp-bcc-hcp phase transformation," Acta Mater. 58(11), 4053-4066 (2010).

${ }^{20} \mathrm{H}$. X. Gao and L.-M. Peng, "Parameterization of the temperature dependence of the Debye-Waller factors," Acta Cryst. A 55, 926-932 (1999).

${ }^{21} \mathrm{H}$. Rauch and D. Petrascheck, "Grundlagen für ein LaueNeutroneninterferometer Teil 1: Dynamische Beugung," AIAU 74405b, Atominstitut der Österreichischen Universitäten, 1976.

${ }^{22} \mathrm{H}$. Rauch and D. Petrascheck, "Dynamical neutron diffraction and its application," in "Neutron Diffraction," edited by H. Dachs (SpringerVerlag, Berlin/Heidelberg, New York, 1978), pp. 303-351.

${ }^{23}$ V. F. Sears, "Scattering lengths for neutrons," in International Tables for Crystallography edited by E. Prince, International Union of Crystallography, Chester, Section 4.4.4 (2006) 444.

${ }^{24} \mathrm{R}$. W. Waschowski, in Landolt-Börnstein, edited by H. Schopper, New Series I/16A (Springer, Berlin, 2000), Chap. 6.

${ }^{25} \mathrm{D}$. Taupin, "Théorie dynamique de la diffraction des rayons $\mathrm{X}$ par les cristaux déformés," Bull. Soc. Franç. Minér. Crist. 87, 469 (1964).

${ }^{26}$ S. Takagi, "A dynamical theory of diffraction for a distorted crystal," J. Phys. Soc. Jpn. 26(5), 1239 (1969).

${ }^{27}$ L. He, A. Dehghan-Manshadi, and R. J. Dippenaar, Mater. Sci. Eng., A 549, 163-167 (2012). 